\title{
Antiviral Therapy for Hepatitis C
}

\author{
Michelle M. Lipman, MD \\ Scott J. Cotler, MD
}

\author{
Address \\ Rush University Medical Center, 1725 West Harrison Street, Suite 206, \\ Chicago, IL 60612, USA. \\ E-mail: michelle_m_lipman@rush.edu \\ Current Treatment Options in Gastroenterology 2003, 6:445-453 \\ Current Science Inc. ISSN 1092-8472 \\ Copyright $\odot 2003$ by Current Science Inc.
}

\section{Opinion statement}

Current treatment for hepatitis $C$ virus infection consists of pegylated interferon and ribavirin. The most important predictors of response to antiviral therapy for HCV include genotype 2 or 3 infection, baseline viral load less than 2 million copies $/ \mathrm{mL}$, and the absence of cirrhosis. Hepatitis C genotype and viral load should be obtained prior to initiating therapy. Liver biopsy can be used to stage the liver disease, to provide prognostic information, and to evaluate for coexisting causes of liver injury. Patients with genotype 1 infection require 48 weeks of therapy and a ribavirin dosage of 1000 to $1200 \mathrm{mg} / \mathrm{d}$ to achieve an optimal response. Patients with genotype 2 or 3 infection require only 24 weeks of treatment and a ribavirin dose of $800 \mathrm{mg} / \mathrm{d}$. Treatment may be discontinued in patients who do not have a 100 -fold reduction in hepatitis $C$ virus RNA level from baseline at week 12 because they are unlikely to achieve a sustained response with further therapy. Patients with cirrhosis and hepatic decompensation or a small hepatocellular carcinoma should be evaluated for liver transplantation.

\section{Introduction}

Hepatitis $\mathrm{C}$ virus (HCV) is estimated to have reached the United States around 1910, based on molecular analysis of long-term serial samples obtained from infected patients [1]. The projected emergence of HCV in the United States coincides with the return of soldiers from the Spanish-American war and the introduction of blood typing to allow for blood transfusion. Currently, HCV is the most common, chronic blood-borne infection in the United States. At least 3.9 million persons are anti-HCV seropositive and 2.7 million have chronic viremia [2].

The rate of chronicity of acute HCV is quite high, with estimates ranging from $54 \%$ to $85 \%$ [3]. Crosssectional studies suggest that cirrhosis develops in $20 \%$ to $25 \%$ of persons with HCV within 20 years, whereas progression to cirrhosis may require 50 or more years in another $25 \%$ to $30 \%$ of infected individuals. The remaining $50 \%$ of persons with HCV appear to have an intermediate rate of development of cirrhosis. More rapid progression of liver disease is associated with alcohol abuse, male gender, older age at the time of infection, and coinfection with HIV [4,5]. Other factors that may accelerate hepatic fibrosis include advancing age, hepatic steatosis, and increased hepatic iron stores. Persons with HCV and cirrhosis develop hepatocellular carcinoma at a rate of $1 \%$ to $4 \%$ per year [6].

Complications and financial costs due to HCV are projected to increase substantially in the coming years based on the large reservoir of infection and the concentration of HCV in young people. Hepatitis $\mathrm{C}$ is the leading indication for liver transplantation, accounting for $40 \%$ to $50 \%$ of cases. Need for liver transplantation due to HCV is expected to continue to rise until 2015, and the demand for repeat transplantation for HCV recurrence is increasing. Direct medical expenditures for HCV are projected to reach $\$ 10.7$ billion between 2010 and 2019, excluding the cost of liver biopsies, hepatocellular carcinoma screening, and antiviral therapy [7].

Hepatitis $C$ virus replication is rapid and error prone, leading to the generation of genetic heterogeneity. Hepatitis $\mathrm{C}$ is categorized into six major genotypes that differ in their amino acid sequence by approximately $30 \%$. Genotype 1 is most prevalent in the United States (75\%), followed by genotype 2 (15\%), and genotype 3 (7\%) [8]. Viral mutations produce closely related strains within an 
individual, which are referred to as quasi-species. Genetic heterogeneity contributes to the high rate of chronicity observed with acute $\mathrm{HCV}$ infection and poses a barrier to vaccine development.

Antibody testing using an enzyme-linked immunosorbent assay (ELISA) is the first step in establishing the diagnosis of hepatitis C. Molecular testing is used for early detection of acute infection, to establish the presence of viremia in chronically infected anti-HCV seropositive persons, and to monitor the response to therapy. Both qualitative and quantitative polymerase chain reaction (PCR) assays are available, and the quantitative tests were recently standardized to international units. Genotype testing should be performed on HCV-treatment candidates. Genotype is the strongest predictor of response to antiviral therapy and guides the duration of treatment. Liver biopsy is the most accurate way to stage HCV-related liver disease, and histologic findings provide prognostic information. Liver biopsy is also important when laboratory tests suggest a coexisting process such as autoimmune hepatitis, iron overload, nonalcoholic steatohepatitis (NASH), or the possibility of cirrhosis. There is a trend toward deferring a pretreatment liver biopsy in patients who are motivated to take treatment and have factors predicting a high sustained virologic response (SVR) rate.

Interferon alfa is the mainstay of treatment for HCV. It has direct antiviral and immune modulating effects, as well as anti-inflammatory and antiproliferative activity. Viral kinetic modeling has helped to characterize the response of HCV to interferon therapy. HCV levels decrease exponentially 24 to 48 hours after initiation of antiviral therapy. The rapid "first phase" decline in viremia is followed by a more gradual "second phase" response with continued treatment [9]. Based on mathematical modeling, Neumann et al. [9] suggested that the first phase decline in viremia is related to interferon blocking HCV production or release. The second phase reduction in virus levels was hypothesized to reflect immune-mediated clearance of infected hepatocytes and inhibition of viral production. Both phases of the viral response are associated with SVR.

Ribavirin is now part of the standard treatment regimen for HCV and is given in combination with interferon. It is a synthetic guanosine nucleoside analogue that reduces aminotransferase levels in patients with HCV, but has a minimal effect on viremia. However, combining ribavirin with interferon improves the antiviral efficacy over interferon alone $[10,11]$. The mechanism of ribavirin in the treatment of HCV remains under investigation. Ribavirin may act in part as an immune modulator and may function as a viral RNA mutagen [12]. Ribavirin inhibits inosine monophosphate dehydrogenase and may reduce viral protein synthesis by decreasing the intracellular concentration of guanosine triphosphate (GTP).

\section{Treatment}

\section{Diet and lifestyle}

- Excess alcohol consumption is associated with an increased rate of progression to cirrhosis. The question is how much alcohol is too much in persons with HCV? A study of $233 \mathrm{HCV}$ patients, $80 \%$ of whom reported consuming less than $140 \mathrm{~g} /$ wk of alcohol, showed that even relatively low alcohol intake is associated with increased levels of viremia and hepatic fibrosis [13]. Ongoing alcohol use decreases the efficacy of treatment for HCV. Abstinence from alcohol is generally recommended for persons with HCV.

- Nonalcoholic steatohepatitis is the most common cause of liver disease in the United States and may contribute to the progression of hepatic fibrosis in persons with HCV. Factors linked to NASH, including obesity (body mass index $>30 \mathrm{~kg} / \mathrm{m}^{2}$ ), steatosis, and diabetes, have been associated with increased hepatic fibrosis in chronic HCV. Type 2 diabetes mellitus and steatohepatitis were recently identified as independent risk factors for advanced liver disease in HCV infection. Response rates to pegylated interferon and ribavirin are reduced in patients with body weights in excess of 75 to $85 \mathrm{~kg}$. Treatment of NASH through weight loss and careful management of diabetes and hyperlipidemia may be beneficial in patients with HCV.

- The relationship between tobacco use and the severity of hepatic fibrosis in HCV was evaluated in a cross-sectional study of 310 patients [14]. Smokers were more often young, male, had consumed alcohol, and had a history of intravenous drug use. However, both age-adjusted and multivariate analysis showed a relationship between tobacco use and increased hepatic fibrosis. Patients should be informed of the potential deleterious effect of tobacco use on the natural history of HCV, and smoking cessation should be strongly encouraged. 
- Increased hepatic iron stores have been linked to more rapid progression of fibrosis. A recent study demonstrated a higher incidence of cirrhosis in patients with HCV who were heterozygous for hereditary hemochromatosis [15]. Persons with HCV should be cautioned against self-medicating with iron and should avoid taking high doses of vitamin $\mathrm{C}$, which can increase iron absorption.

\section{Pharmacologic treatment}

- The primary goal of pharmacologic treatment is to achieve viral eradication. An SVR is defined as HCV RNA undetectable in blood 6 months after completion of antiviral therapy. Over $95 \%$ of patients who achieve an SVR remain free of detectable viremia during 4 to 10 years of follow-up [16,17].

- The secondary goal of pharmacologic treatment is to stabilize or improve liver histology and to reduce the risk of hepatic decompensation and hepatocellular carcinoma. Sustained responders and even some relapsers and nonresponders may attain these secondary benefits from interferon therapy.

Interferon alfa

Standard dosage Monotherapy (now rarely used): 3 million IU of interferon alfa $2 \mathrm{a}$ or $2 \mathrm{~b}$ or $9 \mu \mathrm{g}$ of interferon alfacon-1, three times per week, administered subcutaneously (SC) for 48 weeks. Combination with ribavirin: unmodified interferon is given according to the same dosing schedule when combined with ribavirin. The duration of treatment is based on the HCV genotype and the interim response. Patients with genotype 2 or 3 infection are treated for 24 weeks and those with genotype 1 infection require 48 weeks of therapy.

Contraindications Hypersensitivity to interferon alfa or any component of the product, decompensated liver disease, and autoimmune hepatitis. Use with caution in patients with thrombocytopenia or leukopenia, history of severe depression or other psychiatric illness, severe renal disease, symptomatic heart disease, seizure disorders, or compromised central nervous system (CNS) function.

Main drug interactions Interferon interacts with drugs metabolized by the cytochrome P-450 (CYP) 1A2. Theophylline and phenobarbital levels may increase with interferon administration. Giving interferon with zidovudine increases bone marrow suppression. Interferon may potentiate the neurotoxicty of vidarabine. Vinblastine may increase the toxicity of interferon.

Main side effects See Table 1.

Special points Discontinuation is based on interim response. Treatment is discontinued in patients with detectable viremia at week 12 of monotherapy or at week 24 of interferon and ribavirin therapy in patients with genotype 1 because the SVR is negligible in such cases with continued therapy.

Efficacy The combination of interferon alfa- $2 b$ plus ribavirin significantly increased the number of patients achieving an SVR from $13 \%$ with 48 weeks of therapy with interferon alone to approximately $40 \%$ with the addition of ribavirin [10].

Cost effectiveness Interferon alfa-2b, three million units three times per week SC with ribavirin 1000 $\mathrm{mg}$ (patients less than $<75 \mathrm{~kg}$ ) or $1200 \mathrm{mg}$ (patients $>75 \mathrm{~kg}$ ) daily for 48 weeks costs $\$ 19,303.68$. Younossi et al. [18] determined that the approach of using interferon with ribavirin for all patients was associated with a cost of $\$ 34,792$ and 15.31 quality-adjusted life years (QALYs). They also found that using genotype to guide the duration of therapy was the most cost-effective approach, with an incremental cost-effectiveness ratio of $\$ 7500$ per QALY.

Peginterferon alfa-2b

Standard dosage For monotherapy: $1 \mu \mathrm{g} / \mathrm{kg} \mathrm{SC}$ weekly for 48 weeks. Combination with ribavirin: 1.5 $\mu \mathrm{g} / \mathrm{kg} \mathrm{SC}$ weekly. Patients with genotype 2 or 3 infection are treated for 24 weeks and those with genotype 1 require 48 weeks of therapy.

Contraindications See interferon alfa.

Main drug interactions See interferon alfa. 
Table 1. Common side effects of interferon, pegylated interferon alfa-2a, and pegylated interferon alfa-2b

Flu-like symptoms
Headache
Fatigue
Myalgia
Arthralgia
Musculoskeletal pain
Fever
Chills
Gastrointestinal
Nausea
Anorexia
Diarrhea
Abdominal pain
Hematologic
Neutropenia
Thrombocytopenia
Anemia
Neuropsychiatric
Anxiety
Depression
Insomnia
Irritability
Endocrine
Thyroid dysfunction
Injection site reaction
Alopecia
Rash
Dermatologic
Ans

Main side effects See Table 1.

Special points Neutropenia and thrombocytopenia are dose-limiting toxicities, and blood counts should be monitored closely. Compared with the combination of unmodified interferon and ribavirin, peginterferon alfa- $2 b$ and ribavirin were associated with more flu-like symptoms and more injection site reactions. Treatment is discontinued if HCV RNA remains detectable at week 24.

Efficacy A large registry trial compared treatment with peginterferon alfa- $2 \mathrm{~b}(1.5 \mu \mathrm{g} / \mathrm{kg} / \mathrm{wk})$ and ribavirin $(800 \mathrm{mg} / \mathrm{d}$ ) with unmodified interferon alfa- $2 \mathrm{~b}$ (3 million units three

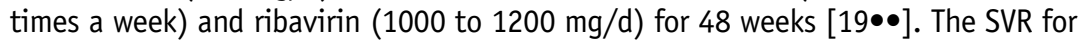
the peginterferon alfa-2b group (54\%) was significantly higher than the SVR in the unmodified-interferon group (47\%). Patients with genotype 1 infection benefited from peginterferon alfa-2b/ribavirin (SVR 42\%) compared with unmodified interferon/ ribavirin (SVR 33\%). Further subset analysis showed that the advantage of peginterferon/ribavirin in genotype 1 was limited to patients with a low baseline viral load $\left(<2 \times 10^{6}\right.$ copies $\left./ \mathrm{mL}\right)$. Patients in both treatment groups with genotype 2 and 3 had an SVR of approximately $80 \%$. A post hoc analysis showed that ribavirin doses of 10.6 to $13 \mathrm{mg} / \mathrm{kg}$ were associated with higher SVRs.

Cost effectiveness Peginterferon alfa-2b (PEG-Intron; Schering, Kenilworth, NJ) $1.5 \mu \mathrm{g} /$ week SC is $\$ 16,363.20$ for 48 weeks of therapy; in combination with ribavirin (Rebetol; Schering, Kenilworth, NJ) 1000 to $1200 \mathrm{mg} / \mathrm{d}$ orally for 48 weeks, the cost is $\$ 34,165$ to $\$ 37,725.36$. Compared with unmodified interferon plus ribavirin, peginterferon alfa-2b plus weight-based ribavirin had an incremental costeffectiveness ratio of $\$ 7378$ per QALY, although the higher cost was offset by projected reductions in future complications of liver disease [20]. 


\author{
Standard dosage Monotherapy: $180 \mu \mathrm{g}$ SC weekly for 48 weeks. Combination therapy with ribavirin: \\ $180 \mu \mathrm{g}$ SC once weekly for 24 weeks in patients with genotype 2 or 3 . Patients \\ with genotype 1 infection require 48 weeks of therapy. \\ Contraindications See interferon alfa. \\ Main drug interactions See interferon alfa. \\ Main side effects See Table 1. \\ Special points Neutropenia and thrombocytopenia are dose-limiting toxicities and blood counts \\ should be monitored closely. The combination of peginterferon alfa-2a and ribavi- \\ rin is associated with less flu-like symptoms and less depression than unmodified \\ interferon and ribavirin. Treatment should be discontinued in patients who do not \\ have a 100-fold reduction or undetectable HCV RNA at treatment week 12. \\ Efficacy A large registry trial compared 48 weeks of peginterferon alfa-2a (180 $\mu \mathrm{g} /$ week) \\ plus ribavirin (1000 to $1200 \mathrm{mg} / \mathrm{d}$ ) with 48 weeks of unmodified interferon alfa-2b

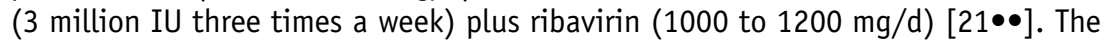 \\ peginterferon alfa-2a/ribavirin group had a significantly higher overall SVR $(56 \%$ \\ vs $44 \%$ ) and a higher SVR in patients with genotype 1 infection (46\% vs $36 \%$ ). \\ Peginterferon alfa-2a/ribavirin showed an advantage over unmodified interferon/ \\ ribavirin in genotype 1 patients with a high viral load (41\% vs 33\%). The SVR \\ with peginterferon alfa-2a/ribavirin in genotype 2 and 3 infection (76\%) was \\ significantly better than unmodified interferon/ribavirin (61\%). \\ Cost effectiveness Peginterferon alfa-2a (Pegasys; Hoffman-La Roche, Nutley, NJ) $180 \mu \mathrm{g} / \mathrm{wk}$ SC for \\ 48 weeks costs $\$ 16,761.60$. In combination with ribavirin (Copegus; Hoffman-La \\ Roche, Nutley, NJ), $1000 \mathrm{mg} / \mathrm{d}$ (patients $<75 \mathrm{~kg}$ ) or $1200 \mathrm{mg} / \mathrm{d}$ (patients $>75 \mathrm{~kg}$ ) \\ for 48 weeks costs $\$ 27,395.40$.
}

\title{
Ribavirin
}

Standard dosage For genotype 1, the dosage is $1000 \mathrm{mg} / \mathrm{d}$ in patients weighing less than $75 \mathrm{~kg}$ and $1200 \mathrm{mg} / \mathrm{d}$ for patients weighing more than $75 \mathrm{~kg}$, administered orally in two divided doses. For genotype 2 or $3,800 \mathrm{mg} / \mathrm{d}$ of ribavirin is the appropriate dosage.

Contraindications Patients with renal insufficiency with creatinine clearance less than $50 \mathrm{~mL} / \mathrm{min}$. Ribavirin is teratogenic and neither women nor men should conceive a child within 6 months of taking this medication. Use of two forms of birth control is indicated.

Main drug interactions Antacids (containing calcium, magnesium, or simethicone ) may decrease the absorption of ribavirin. The combination of ribavirin and dideoxyinosine can lead to mitochondrial dysfunction with the development of pancreatitis and lactic acidosis.

Main side effects Hemolytic anemia, which can be particularly severe in patients with renal insufficiency who have reduced ribavirin clearance. Combining ribavirin with interferon increases the frequency of anorexia, nausea, dyspnea, insomnia, pruritus, and rash compared with that observed with interferon alone.

Special points Ribavirin has minimal activity against HCV when given as monotherapy and should be used in combination with interferon.

Cost effectiveness Rebetol (used in conjunction with PEG-Intron) 1000 to $1200 \mathrm{mg} / \mathrm{d}$ orally for 48 weeks costs $\$ 17,801.80$ to $\$ 21,362.16$. Copegus (Hoffman-La Roche, Nutley, NJ), which is used in conjunction with Pegasys, in dosages of 1000 to $1200 \mathrm{mg} / \mathrm{d}$ orally for 48 weeks costs $\$ 10,633.80$.

\section{Treatment in special situations}

Interferon is highly effective in treating acute HCV, with an SVR of $98 \%$ in one study [22]. A meta-analysis of the limited information available found that delaying therapy by 2 to 4 months does not compromise the probability of a favorable response to interferon and might avoid treatment of persons who will spontaneously clear the acute infection [23]. 
The efficacy of maintenance interferon therapy in changing the natural history of $\mathrm{HCV}$ in nonresponding patients is unproven, although data are accumulating to suggest that this strategy may be beneficial. In one report, one third of virologic nonresponders with bridging fibrosis or cirrhosis had an improvement in hepatic inflammation from baseline that persisted for 6 months after the completion of peginterferon alfa-2a therapy [24]. Other studies suggest that interferon may stabilize fibrosis, even in nonresponders [25], and that fibrosis can regress in sustained responders [26]. Retrospective analyses indicate that interferon therapy may reduce the risk of hepatocellular carcinoma [27]. A recent study demonstrated a survival benefit in patients with HCV and hepatocellular carcinoma treated with interferon after tumor ablation by ethanol injection over those who received ethanol injection alone [28]. Ribavirin is generally omitted from maintenance regimens due to its limited effect on liver histology and added side effects. The Hepatitis C Antiviral Long-term Treatment against Cirrhosis (HALT-C) study is an ongoing National Institutes of Health-sponsored multicenter trial designed to evaluate the effect of maintenance pegylated interferon on progression to cirrhosis, hepatic decompensation, and hepatocellular carcinoma in patients with advanced HCV.

Liver transplantation for hepatitis $C$

Hepatitis C-related cirrhosis is the most common indication for liver transplantation, accounting for $40 \%$ or more of liver transplants in the United States. The infection persists after the transplant in nearly all patients, and $8 \%$ to $30 \%$ of patients progress to cirrhosis within 5 to 7 years of transplantation $[29,30]$. A recent study of United Network for Organ Sharing registry data from 11,036 patients who received transplants between 1992 and 1998 showed reduced patient and allograft survival in HCV-positive versus HCV-negative cases [31].

Management of immunosuppression might impact on the natural history of recurrent HCV. A retrospective analysis led one group to suggest a possible benefit for very gradual tapering of corticosteroid after the transplant in an attempt to avoid the development of a severe recurrent hepatitis [32]. Bolus corticosteroids should be avoided in patients with HCV who have equivocal changes of rejection due to an association between intense immunosuppression and the development of progressive HCV.

Patients with decompensated cirrhosis on the liver transplant list are generally debilitated and frequently have cytopenias and ascites, making it difficult to administer interferon therapy. Initiating interferon in the early post-transplant period was found to be effective in delaying the onset of histologic recurrence, although it did not prevent the recurrence of HCV viremia [33,34].

Interferon and ribavirin initiated more than 3 months after transplantation led to SVRs of $5 \%$ to $27 \%$ in small studies of carefully selected patients [35-37]. Ribavirin-associated anemia was common in these trials, and dropout rates of approximately $20 \%$ to $40 \%$ due to adverse effects were reported. Studies are underway to evaluate the safety and efficacy of pegylated interferon and ribavirin for the treatment of HCV in liver transplant recipients.

Many transplant centers now accept livers from HCV-positive donors for transplantation into HCV-infected recipients to increase the donor organ supply. A comparison of outcomes in patients with HCV who received infected allografts and those who received livers from uninfected donors showed no difference in post-transplant histologic progression or 4-year graft or patient survival [38].

Other treatments

Silymarin (milk thistle)

Silymarin (milk thistle), an extract of Silybum marianum, contains multiple biologically active compounds, including the flavonoid silibinin. It acts as an antioxidant and a free radical scavenger. Silymarin prevents glutathione depletion and free radical formation and may have antifibrotic activity. There are no randomized, controlled trials assessing silymarin for the treatment of HCV. In small, uncontrolled studies, silymarin therapy decreased alanine aminotransferase levels but did not lower HCV levels. 
Lactoferrin is a milk protein belonging to the iron transporter family with antiviral activity against herpes simplex virus-1, cytomegalovirus, and HIV. Other data suggest that lactoferrin might have immune-modulating and antiproliferative effects. Bovine lactoferrin was found to have activity against HCV in an in vitro model and was shown to bind to the HCV E1 and E2 proteins [39]. Lactoferrin is being studied as a potential treatment for HCV.

\section{Emerging therapies}

\section{Protease inhibitors}

The success of protease inhibitors for the treatment of HIV has led to intense interest in developing HCV-specific protease inhibitors. The NS3 serine protease offers three targets, which include the substrate binding site, the zinc cofactor that provides structural support to the peptide, and the point of interaction between NS3 and NS4A. A number of different NS3 inhibitors are in development. A phase 1 trial of the orally administered protease inhibitor BILN 2061 showed dramatic reductions in HCV RNA levels with 48 hours of therapy [40].

Interferon $\gamma$

Interferon $\gamma$ is produced by activated T lymphocytes and natural killer cells and has antiviral, antifibrotic, and immune-modulating effects. A phase II study is underway to evaluate whether recombinant interferon $\gamma$-1b can reduce hepatic fibrosis in patients with advanced histologic changes caused by HCV. Furthermore, interferon $\gamma$ was shown to inhibit RNA replication and protein synthesis in subgenomic HCV replicon constructs [41]. Preliminary in vitro data suggest that interferon $\gamma$ and interferon alfa may be synergistic in their antiviral activity, leading to interest in combining these agents for the treatment of HCV.

\section{Polymerase inhibitors}

RNA polymerase is required for synthesis of both negative-stranded RNA intermediate and positive-stranded genomic HCV RNA. Hepatitis C polymerase inhibitors, including nucleoside analogues, non-nucleoside analogues, and pyrophosphate analogues, have been identified. Several of these agents are being developed for clinical use.

\section{Ribozymes}

Ribozymes are catalytic RNA molecules that cleave specific RNA sequences. An HCV specific ribozyme significantly inhibited viral replication in a dosedependent fashion in cell culture. However, toxicity and limited clinical efficacy led to discontinuation of a phase II study of ribozyme therapy for HCV.

Small interfering RNA

Small interfering RNA are double-stranded RNAs that downregulate virtually any gene at the post-translational level through destruction of a specifically targeted mRNA. Development of small interfering RNA molecules with activity against HCV is in progress.

Levovirin is the L-sugar analogue of ribavirin that appears to have immunomodulatory activity. It does not cause the hemolytic anemia observed with ribavirin therapy. A phase 1 study demonstrated that levovirin was orally absorbed and well tolerated. A proof-of-concept study with pegylated interferon is planned. Viramidine is the amidine version of ribavirin that is converted to ribavirin during first-pass metabolism and concentrated in the liver. A proof-of-concept study with pegylated interferon is in progress. 
Papers of particular interest, published recently, have been highlighted as:

- Of importance

$\bullet \quad$ Of major importance

1. Tanaka Y, Hanada K, Mizokami M, et al.: A comparison of the molecular clock of hepatitis $\mathrm{C}$ virus in the United States and Japan predicts that hepatocellular carcinoma incidence in the United States will increase over the next two decades. Proc Natl Acad Sci U S A 2002, 99:15584-15589.

2. Alter MJ, Kruszon-Moran D, Nainan OV, et al.: The prevalence of hepatitis $C$ virus infection in the United States, 1988 through 1994. N Engl J Med 1999, 341:556-562.

3. Seeff L: Natural history of chronic hepatitis C. Hepatology 2002, 36:S35-S36.

4. Poynard T, Bedossa P, Opolon P: Natural history of liver fibrosis progression in patients with chronic hepatitis C. Lancet 1997, 349:825-832.

5. Benhamou $Y$, Bochet $M$, Di Martino V, et al.: Liver fibrosis progression in human immunodeficiency virus and hepatitis $C$ virus coinfected patients. Hepatology 1999, 30:1054-1058.

6. El-Serag HB: Hepatocellular carcinoma and hepatitis $\mathrm{C}$ in the United States. Hepatology 2002, 36:S74-S83.

7. Wong JB, McQuillan GM, McHutchison JG, et al: Estimating future hepatitis $\mathrm{C}$ morbidity, mortality, and costs in the United States. Am J Public Health 2000, 90:1562-1569.

8. Hoofnagle JH: Course and outcome of hepatitis C. Hepatology 2002, 36:S21-S29.

9. Neumann AU, Lam NP, Dahari H, et al.: Hepatitis C viral dynamics in vivo and the antiviral efficacy of interferon-alpha therapy. Science 1998, 282:103-107.

10. McHutchison JG, Gordon SC, Schiff ER, et al.: Interferon alfa-2b alone or in combination with ribavirin as initial treatment for chronic hepatitis C. N Engl J Med 1998, 339:1485-1492.

11. Poynard T, Marcillin P, Lee SS, et al.: Randomised trial of interferon alfa $2 \mathrm{~b}$ plus ribavirin for 48 weeks or for 24 weeks versus interferon alfa $2 \mathrm{~b}$ plus placebo for 48 weeks for treatment of chronic infection with hepatitis C virus. Lancet 1998, 352:1426-1432.

12. Crotty S, Maag D, Arnold JJ, et al.: The broad-spectrum antiviral ribonucleoside ribavirin is an RNA virus mutagen. Nat Med 2000, 12:1375-1379.

13. Pessione F, Degos F, Marcellin P, et al.: Effect of alcohol consumption on serum hepatitis $C$ virus RNA and histological lesions in chronic hepatitis C. Hepatology 1998, 27:1717-1722.

14. Pessione F, Ramond MJ, Njapoum C, et al.: Cigarette smoking and hepatic lesions in patients with chronic hepatitis C. Hepatology 2001, 34:121-125.

15. Tung B, Emond M, Bronner M, et al.: Hepatitis C, iron status, and disease severity: relationship with HFE mutations. Gastroenterology 2003, 124:318-326.
16. Marcellin P, Boyer N, Gervais A, et al.: Long-term histologic improvement and loss of detectable intrahepatic HCV RNA in patients with chronic hepatitis $\mathrm{C}$ and sustained response to interferon-alpha therapy. Ann Intern Med 1997, 127:875-881.

17. Lau DT, Kleiner DE, Ghany MG, et al: : 10-Year follow-up after interferon-alpha therapy for chronic hepatitis C. Hepatology 1998, 28:1121-1127.

18. Younossi Z, Singer M, McHutchison J, Shermock K: Cost effectiveness of interferon alfa2b combined with ribavirin for the treatment of chronic hepatitis $\mathrm{C}$. Hepatology 1999, 30:1318-1324.

$19 . \bullet$ Manns MP, McHutchison JG, Gordon SC, et al.: Peginterferon alfa-2b plus ribavirin compared with interferon alfa-2b plus ribavirin for initial treatment of chronic hepatitis C: a randomised trial. Lancet 2001, 358:958-965.

This randomized trial compared peginterferon alfa- $2 b$ plus ribavirin with interferon alfa-2b plus ribavirin, changing the standard of care for the management of hepatitis $\mathrm{C}$ to pegylated interferon and ribavirin. Peginterferon alfa-2b plus ribavirin significantly improved the rate of sustained virologic response over interferon alfa-2b and ribavirin.

20. Siebert U, Sroczynski G, Rossol S, et al.: Cost effectiveness of peginterferon alpha-2b plus ribavirin versus interferon alpha- $2 b$ plus ribavirin for initial treatment of hepatitis C. Gut 2003, 52:425-432.

21.• Fried M, Shiffman M, Reddy R, et al.: Peginterferon alfa-2a plus ribavirin for chronic hepatitis $\mathrm{C}$ virus infection. N Engl J Med 2002, 347:975-982.

This randomized trial compared peginterferon alfa-2a plus ribavirin with interferon alfa-2b plus ribavirin for the initial treatment of chronic hepatitis C. It demonstrated that onceweekly peginterferon alfa-2a plus ribavirin was well tolerated and produced significant improvements in the rate of sustained virologic response rates.

22. Jaeckel E, Cornberg M, Wedemeyer $\mathrm{H}$, et al.: Treatment of acute hepatitis $\mathrm{C}$ with interferon alfa-2b. N Engl J Med 2001, 345:1452-1457.

23. Alberti A, Boccato S, Vario A, Benvegnu L: Therapy of acute hepatitis C. Hepatology 2002, 36:S195-S200.

24. Heathcote EJ, Shiffman ML, Cooksley GE, et al.: Peginterferon alfa-2a in patients with chronic hepatitis C and cirrhosis. N Engl J Med 2000, 343:1673-1680.

25. Sobesky R, Mathurin P, Charlotte F, et al.: Modeling the impact of interferon alfa treatment on liver fibrosis progression in chronic hepatitis $\mathrm{C}$ : a dynamic view. Gastroenterology 1999, 116:378-386.

26. Poynard T, McHutchison J, Davis GL, et al.: Impact of interferon alfa- $2 b$ and ribavirin on progression of liver fibrosis in patients with chronic hepatitis $\mathrm{C}$. Hepatology 2000, 32:1131-1137. 
27. Yoshida $H$, Shiratori $Y$, Moriyama M, et al.: Interferon therapy reduces the risk for hepatocellular carcinoma: national surveillance program of cirrhotic and noncirrhotic patients with chronic hepatitis $\mathrm{C}$ in Japan. Ann Intern Med 1999, 131:174-181.

28. Shiratori $Y$, Shiina $S$, Teratani $T$, et al: Interferon therapy after tumor ablation improves prognosis in patients with hepatocellular carcinoma associated with hepatitis C virus. Ann Intern Med 2003, 138:299-305.

29. Prieto $M$, Berenguer M, Rayon JM, et al.: High incidence of allograft cirrhosis in hepatitis $\mathrm{C}$ virus genotype $1 \mathrm{~b}$ infection following transplantation: relationship with rejection episodes. Hepatology 1999, 29:250-256.

30. Gane EJ, Portmann BC, Naoumov NV, et al.: Long-term outcome of hepatitis $\mathrm{C}$ infection after liver transplantation. N Engl J Med 1996, 334:815-820.

31. Forman LM, Lewis JD, Berlin JA, et al.: The association between hepatitis $\mathrm{C}$ infection and survival after orthotopic liver transplantation. Gastroenterology 2002, 122:889-896.

32. Brillanti S, Vivarelli M, De Ruvo N, et al.: Slowly tapering off steroids protects the graft against hepatitis $\mathrm{C}$ recurrence after liver transplantation. Liver Transpl 2002, 8:884-888.

33. Singh NG, Wannstedt CF, Shakil AO, et al.: Interferonalpha for prophylaxis of recurrent viral hepatitis $\mathrm{C}$ in liver transplant recipients. Transplantation 1998, 65:82-86.

34. Sheiner PA, Boros P, Klion FM, et al.: The efficacy of prophylactic interferon alfa- $2 b$ in preventing recurrent hepatitis $\mathrm{C}$ after liver transplantation. Hepatology 1998, 28:831-838.
35. Ahmed J, Dodson SF, Demetris AJ, et al.: Recurrent hepatitis $\mathrm{C}$ after liver transplantation: a nonrandomized trial of interferon alfa alone versus interferon alfa and ribavirin. Liver Transpl 2001, 7:863-869.

36. Alberti AB, Belli LS, Airoldi A, et al.: Combined therapy with interferon and low-dose ribavirin in posttransplantation recurrent hepatitis C: a pragmatic study. Liver Transpl 2001, 7:870-876.

37. Shakil AO, McGuire B, Crippin JS, et al.: A pilot study of interferon-alfa and ribavirin combination in liver transplant recipients with recurrent hepatitis C. Hepatology 2002, 36:1253-1258.

38. Testa G, Goldstein RM, Netto G, et al:: Long-term outcome of patients transplanted with livers from hepatitis C-positive donors. Transplantation 1998, 65:925-929.

39. Ikeda M, Sugiyama K, Tanaka $T$, et al.: Lactoferrin markedly inhibits hepatitis $\mathrm{C}$ virus infection in cultured human hepatocytes. Biochem Biophys Res Commun 1998, 245:549-553.

40. Benhamou Y, Hinrichsen H, Sentjens R, et al:: Safety, tolerability and antiviral effect of BILN 2061, a novel HCV serine protease inhibitor, after oral treatment over 2 days in patients with chronic hepatitis $\mathrm{C}$, genotype 1, with advanced liver fibrosis [abstract]. Hepatology 2002, 36:563.

41. Frese $\mathrm{M}$, Schwarzle V, Barth $\mathrm{K}$, et al.: Interferon-gamma inhibits replication of subgenomic and genomic hepatitis C virus RNAs. Hepatology 2002, 35:694-703. 